\title{
Using Telehealth as a Model for Blockchain HIT Adoption
}

Brennan Bennett

Editor's note: This month, Telehealth and Medicine Today brings to its readers the second in a series authored by Brennan Bennett on telehealth and blockchain technology. In this article, the focus is on their shared core philosophy of empowering the individual.

Telemedicine and blockchain technology share a core philosophy of empowering the individual. Blockchain solutions that focus on empowering patients and enhancing the workflows for the providers who treat them continue to make big headlines, as does enterprise investment and adoption of telehealth. Both models focus on directto-consumer health services, with a personalized care experience designed from the ground up to save time and money for everyone involved. The typical binding factor between the telehealth and HIT (health information technology) blockchain adoption is a patient centric, value-based care model. Therefore, it is as no coincidence that value-based care is at the center of the fastest growing (and operational) part of HIT blockchain adoption. For this reason, telehealth can demonstrate adoption synergies than most other lines of business in healthcare cannot.

Telehealth is used to empower consumers to make better healthcare choices, have higher access care, and lower medical costs by getting help when they need it instead of waiting until it's necessary. Blockchain technology can improve existing technological applications of telemedicine by providing more quality assurance in the data retained each visit. Additionally, blockchain can build incentive structures based on its ability to facilitate an economy of scale (i.e., producing more services at a lower cost). The only thing missing from combining high data integrity with a scalable solution for producing more services at a lower cost is a mechanism for incentivization- enter crypto-currencies, also known as tokens.

\section{Bitcoin and Tokens}

Following the birth of Bitcoin, other alternative currencies, or tokens, arose as people developed blockchain solutions to be less about binary transactions and more about the quality of interaction. To accomplish this, tokens had to take on various kinds of rights that enabled various kinds of actions, or reactions, to be made. These "rights" 
have evolved from only being able to make payments on the network (i.e., payment rights tokens), to innovative functionalities like being rewarded for making a human decision (i.e., contribution rights tokens). Contribution in healthcare is akin to chain of custody of a patient's healthcare data as multiple healthcare professionals review diagnosis, test results, treatment and ultimately discharge plan.

As healthcare systems continue to face roadblocks in the exchange of healthcare data, blockchain technology seemingly offers several solutions for the protection and management of patient information. One of many blockchain startup companies, Patientory, enables patients to secure their own data utilizing blockchain technology. Patientory is not a standalone blockchain EMR (emergency medical record) solution, but it does take a step in the right direction by enabling healthcare consumers to use their personal health data as a resource to securely socialize with multiple health teams and other patients experiencing similar health issues.

Following the footsteps of Patientory is Healthcoin, which utilizes blockchain technology to reduce the effects of diabetes. Another offshoot, Dentacoin, utilizes blockchain based applications to facilitate global dental care. Ultimately, programs like Patientory and Dentacoin are still in their beginning stages and remain a work in progress. The proverbial kinks in the chain-system require repair and whether cryptocurrencies or smart contracts provide a cure-all solution to an evolving health care industry remains to be seen.

Other types of tokens have enhanced their functionality by adding their own coding to the core design. Ethereum for example, implements smart contracts. Smart contracts are self-executing and auto-enforced digital agreements without necessity of a middle-man. Some contractual arrangements involve financial terms and when met, the data-oriented contract releases units or "ether." Patientory and Healthcoin incorporate Ethereum's technology, but have developed their own token to run on the Ethereum blockchain specifically for their respective ecosystem. For example, when using Patientory, a doctor can use the network's native token, PTOY, to get a retrieve a patient's medical history. Dentacoin's smart contract design is slightly different; fill out a survey and in return the amount earned pays for further care at a partnered dental clinic.

\section{A Direct-to-Consumer Philosophy}


Blockchain technology and smart contracts in a sense remove the third-party, empowering more of the direct to consumer philosophy, just like telehealth. Medicine's metamorphosis through integration of modern technologies naturally causes a paradigm shift in clinical practices, and often a divide among professional opinion follows the change. Using cryptocurrencies in healthcare is not immune, as believers opt to reside in either camp. Standardization of any process creates interest groups, and as always, there are advantages and disadvantages to adoption.

Frequent access to telehealth services allows early detection of disease and hence lowers the medical cost for patients, doctors, and insurance companies.

Nonetheless, the efforts to reduce the administrative costs of healthcare are major motivating factors behind the application of telemedicine. For this reason, Blockchain may also provide a glimpse of how future virtual care communities rooted in telemedicine can be self-sustaining by ensuring transparency in addition to redefining trust. Point Nurse has developed a blockchain based member-owner community that gives healthcare professionals an entrepreneurial channel to do what they do best- help people. Their reward, “...credit toward future ownership of the community including a share of any profits." Essentially, healthcare professionals such as LPNs, RNs and even med techs are empowered to build themselves as a brand and connect with patients seeking home health services either in their community or via telehealth.

Blockchain adoption still has a long, long way to go to achieve adoption in the healthcare sector. Telehealth can demonstrate quite clearly how healthcare technology can evolve faster when focused on direct to consumer value based care. Understanding how could lead those developing major HIT blockchain use cases to refine their focuses, even in administrative workflows. 


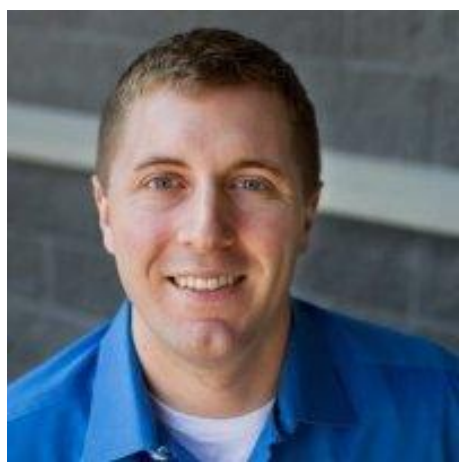

Brennan Bennett is the Founder and Managing Editor of Blockchain Healthcare Review, a publication covering technical innovations in blockchain technology and how it is reshaping the future of health data governance. He is an experienced HIT strategy consultant with a background in the Fortune 100 space. Additionally, Brennan holds a M.S. in Biomedical Informatics from Rutgers-The State University of New Jersey.

Department: Blockchain

Tags: Blockchain, Bitcoin, token, telemedicine, Ethereum, Patientory, Dentacoin, Healthcoin, EMR, security 